\title{
Reaction mechanism of $O$-acylhydroxamate with cysteine proteases
}

\author{
R SHANKAR and P KOLANDAIVEL* \\ Department of Physics, Bharathiar University, Coimbatore 641046 \\ e-mail: ponkvel@hotmail.com
}

MS received 14 June 2007; accepted 31 July 2007

\begin{abstract}
The gas-phase reaction mechanism of $O$-acylhydroxamate with cysteine proteases has been investigated using $a b$ initio and density functional theory. On the irreversible process, after breakdown of tetrahedral intermediate (INT1), small 1-2 anionotropic has been formed and rearranged to give stable by-products sulfenamide (P1) and thiocarbamate (P2) with considerable energy loss. While, on the reversible part of this reaction mechanism, intermediate (INT2) breaks down on oxidation, to form a stable product (P3). Topological and AIM analyses have been performed for hydrogen bonded complex in this reaction profile. Intrinsic reaction coordinates [IRC, minimum-energy path (MEP)] calculation connects the transition state between R-INT1, INT1-P1 and INT1-P2. The products P1, P2 and P3 are energetically more stable than the reactant and hence the reaction enthalpy is found to be exothermic.
\end{abstract}

Keywords. Reaction mechanism; O-acylhydroxamate; cysteine proteases; sulfenamide; thiocarbamate.

\section{Introduction}

One of the biggest challenges in biochemistry is to understand the nature of enzyme reactions at molecular level. ${ }^{1}$ Despite the intensive work over several decades, there is still qualititative understanding of reaction mechanism of enzymes is missing. Proteases are involved in numerous important physiological processes including, digestion, bloodcoagulation, wound healing, fertilization, cell differentiation and growth, cell signalling, and immune response.

Owing to the rapid progress of computational chemistry, understanding of the structure-function relationship of enzyme reactions is becoming an important issue in the theoretical chemistry. The reaction profile of the cysteine proteases inhibitor is also one of the important problems in biochemistry. Proteinases are playing an increasingly important role as a target in the field of drug design. ${ }^{2}$ Many diseases or their symptoms originate from a deficiency or an excess of a specific metabolite, which is either a substrate or product of a proteolytic reaction. ${ }^{4}$ Thus, modulation of a single enzyme in a sequence of reaction steps can influence the overall effect of a physiological enzyme cascade. Therefore, research on enzyme inhibitors is an important approach in medicinal chemistry. ${ }^{3}$ Especially, mechanism-based inhibitor compounds activated during the interaction

\footnotetext{
*For correspondence
}

between target protein and an inhibitor, have potential to lower the side effects of drug administration. Since the reactivity of the inhibitors depends on the catalytic action of the target enzyme on that inhibitor, nonspecific interactions with other protein can be circumvented. ${ }^{4}$ Cysteine proteases are ubiquitous enzymes largely distributed in living organisms and are involved in different important metabolic processes and diseases involving various therapeutic fields, such as, cardiovascular, oncology, osteoporosis, and arthritis.

Till now, twenty one families of cysteine proteases have been discovered ${ }^{5}$, almost half of them are found in viruses and others are found in bacteria, fungi, protozoa and plants. In mammals, two main groups of cysteine proteases are present: cytosolic calpains and lysosomal cathepsins. ${ }^{5,6}$ The cysteine protease inhibitor regulates the action of proteases and plays a significant role in the protection of plants from pest and pathogen attack. The mammalian cysteine proteases are involved in a variety of pathological processes including dysregulated protein turnover such as muscular dystrophy, bone resorption, growth and malignancy of tumors, and myocardial infection. ${ }^{7,8}$ Therefore, these enzymes are promising targets for the development of inhibitors as therapeutic agents. Most of them exhibit a peptide segment for recognition by the enzymes and an electrophilic building block for reaction with the cysteine residue of the enzyme's active site as common structural features. 
Nowadays, number of reversible and irreversible cysteine protease inhibitors has been studied. Among them, $O$-acylhydroxamate is the most effective reversible inhibitor of cysteine proteases, and is therefore important in the field of drug design and medicinal chemistry. In addition to this, numerous groups have made important contributions towards the development of cysteine protease inhibitors. These classes of compounds were first described by Fischer and co-workers ${ }^{9,10}$ as irreversible inhibitor of serine proteinases, such as $\alpha$-chymotrypsin and dipeptidyl peptidase. Latter on, they performed NMR study for inhibition of cysteine proteinase, but they have uncovered the reaction profile. Robinson, et $a l^{8}$ have described ${ }^{13} \mathrm{C}$ and ${ }^{15} \mathrm{~N}$ NMR characterization of the papain adduct obtained from the $O$-acylhydroxamate having a novel sulfonamide structure. Smith, et $a l^{11}$ have reported that cathepsin B inactivation, the putative tetrahedral intermediate could break down to give turnover products or could produce a stable adduct either by (a) migration of the peptidyl group in a manner similar to a loassen re-arrangement ${ }^{12}$ to give a thiocarbamate or by (b) migration of the enzyme thiol group to afford a sulfenamide. Hoffmann and his group ${ }^{13}$ have employed quantum mechanical methods which shed new light on the mechanism of the reaction of azathioprine, with cysteine in aqueous solution, in which the first step in the reaction involves the nucleophilic attack of $\mathrm{COO}^{-}$of cysteine on the $\mathrm{C}$ atom of the imidazole ring of azathioprine. This step is followed by subsequent nucleophilic attack of the $\mathrm{SH}$ group of cysteine on the $\mathrm{C}$ atoms of the imidazole ring, which causes the $\mathrm{COO}^{-}$group to leave. Thus the cysteine acts in the reaction with azathioprine not only as a reactant, but also as a catalyst of the reaction. Therefore, it can be concluded that biogenic thiol, glutathione or cysteine, facilitate the first and crucial step of azathioprine metabolism, due to the presence of $\mathrm{COO}^{-}, \mathrm{SH}$, and $\mathrm{NH}_{3}^{+}$groups in their molecules.

So for, we know a little about the structural features and binding modes of these propeptides to their target cysteine proteases. A better understanding of these aspects could provide valuable informations for the development of potent and highly selective inhibitors. So, the aim of the present study is to calculate the energy barrier for the reaction mechanism of $O$-acylhydroxamate with cysteine proteases by employing B3LYP and B3PW91 functionals of DFT method with $6-31+G^{*}$ basis sets. The local minima are true minima on the potential energy surfaces, and also, transition state structures have also been identi- fied. In addition to that, geometrical parameters for local minima, transition states, and the associated barrier height for all the states have also been determined.

\section{Computational details}

Geometry of molecules at all stationary points in the potential energy surface have been optimized using B3LYP and B3PW91 functionals of density functional theory implementing $6-31+G^{*}$ basis set. Stationary points located have been characterized by computing vibrational frequencies. The nature of the TS has been confirmed not only by the imaginary frequency and also by IRC calculations. ${ }^{14}$ Further more, single point energy calculations have been performed at MP2 level of theory for the optimized geometry of B3LYP/6-31 + G* level of theory. The zero-point vibrational energy corrections (ZPVE) have been made for the energy of B3LYP/6-31 + G* and B3PW91/6-31 + G* levels of theory. The topological parameters, like the charge density $(\rho)$ and its second derivative $\left(\nabla^{2} \rho\right)$ for bonds are calculated using Bader's atoms in molecules (AIM). ${ }^{15}$ The analysis of charge distribution and charge transfer process are performed using NBO 3.1 Program. $^{16}$ All these calculations were performed using Gaussian $98 \mathrm{~W}$ program. ${ }^{17}$ The transition state calculation was performed for intrinsic reaction coordinates (IRC, minimum-energy path (MEP)). The IRC calculation examines the reaction path leading down from a transition structure on a potential energy surface. The calculation starts at the saddle point and follows the reaction in both directions. The IRC calculations definitely connect two minima on the potential energy surface by a path passing through the transition state between them. However, two minima on a potential energy surface may have more than one reaction path connecting them, corresponding to different transition structure through which the reaction passes. ${ }^{18}$

\section{Results and discussion}

A detailed search of potential energy surface (PES) for the $O$-acylhydroxamate with cysteine proteases reaction mechanism has been performed using DFT method and single point calculations were performed at MP2 level of theory using DFT geometries. The stable structure corresponding to energy minima is identified using frequency analysis and the transi- 

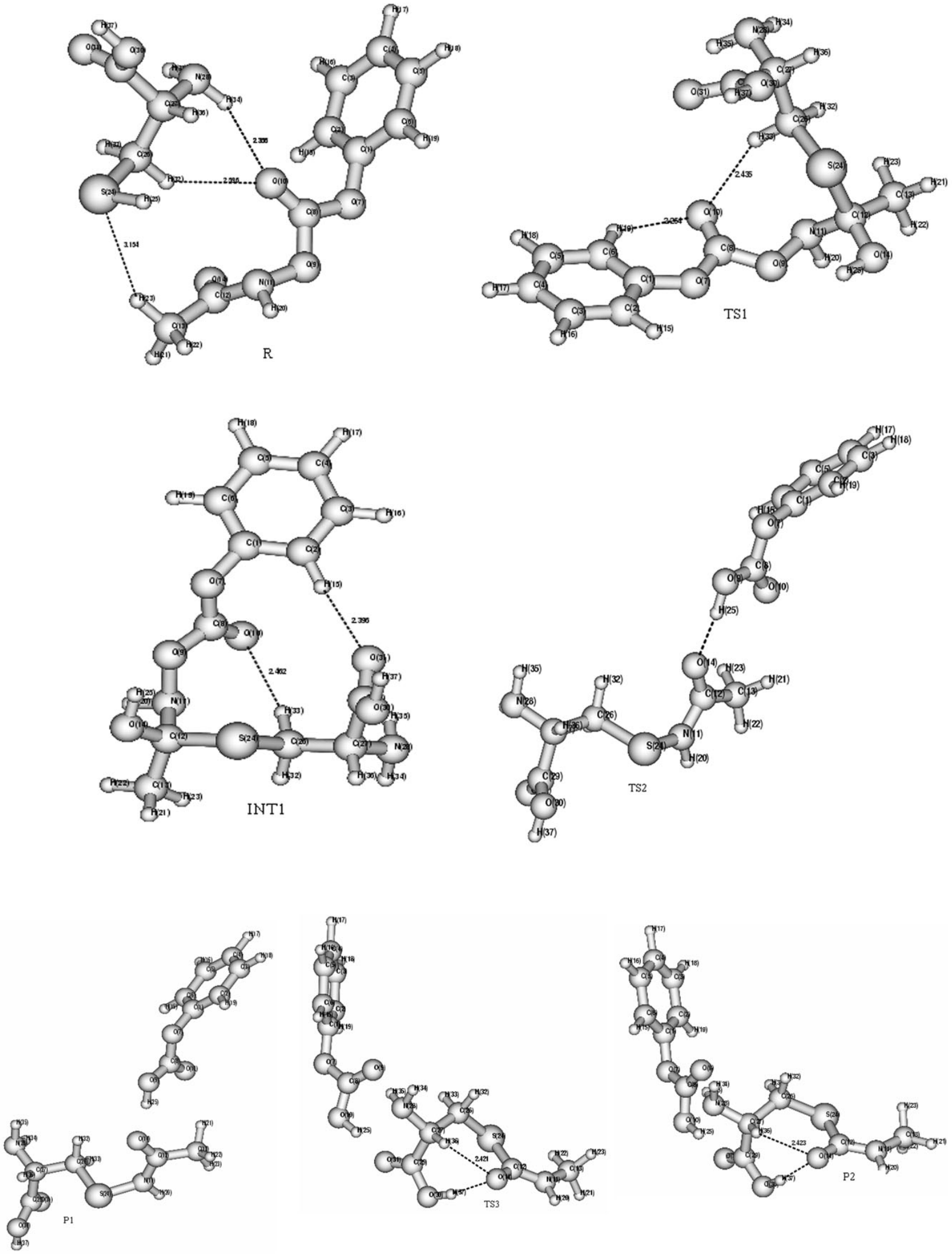

Figure 1. (contd...) 

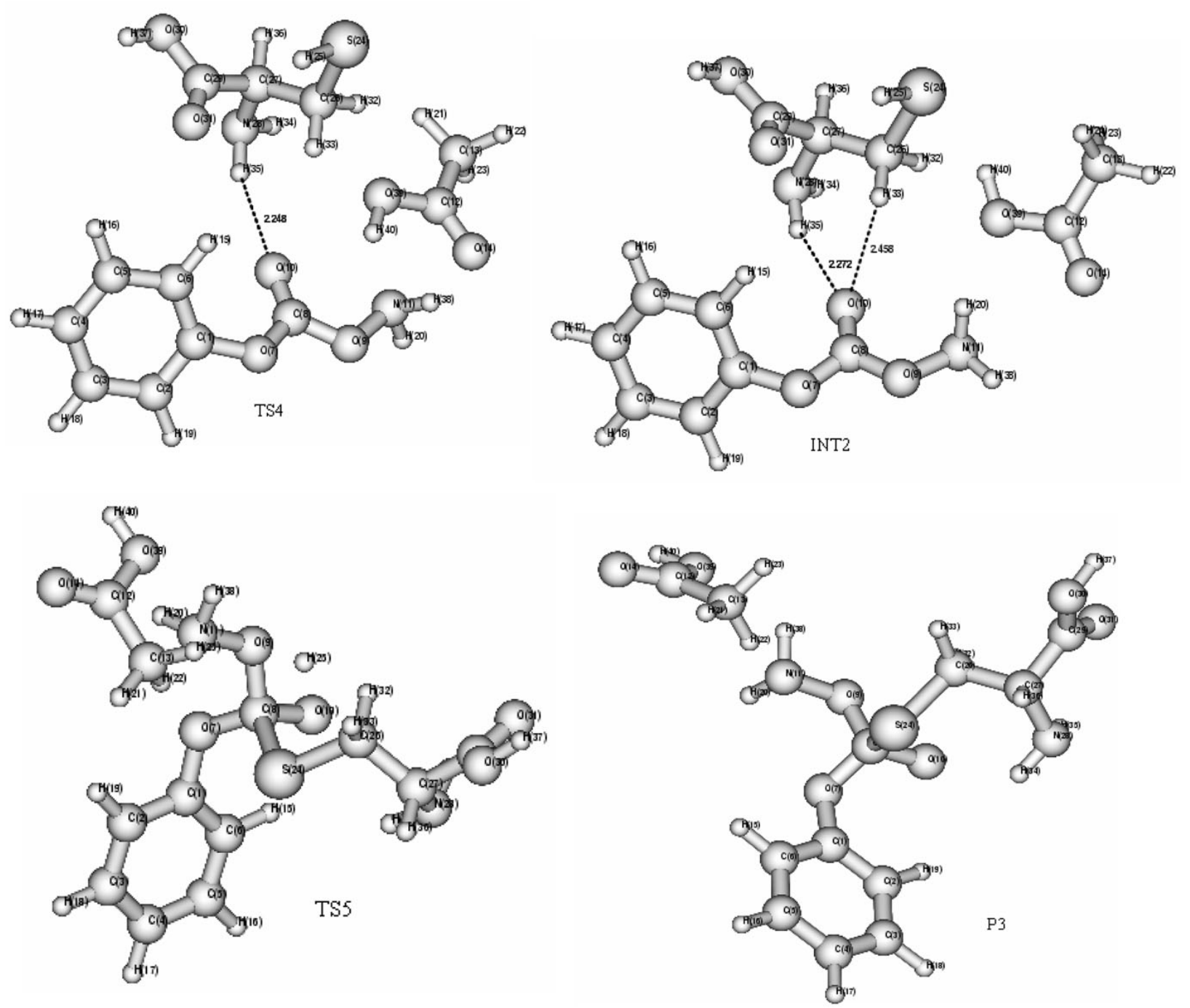

Figure 1. Optimized electronic structures of R, TS1, INT1, TS2, P1, TS3, P2, TS4, INT2, TS5, and P3 for the reaction mechanism of $\mathrm{O}$-acylhydroxamate with cysteine proteases.

tion state structure is characterized as first order saddle points by calculating Hessian matrix. The zero point vibrational energy corrections have been made in order to predict the accurate and reliable energy. The electronic structures of the reactant $\mathrm{R}$, transition states (TS1, TS2, TS3, TS4, and TS5), the intermediates (INT1, and INT2) and products (P1, P2 and P3) which are depicted in figure 1 and the corresponding geometrical parameters are presented in tables 1-3. The (IRC) calculations connect the transition state between R-INT1, INT1-P1 and INT1$\mathrm{P} 2$, but it fails to converge at transition state between the INT1-INT2 and INT2-P3.

\subsection{Formation of the tetrahedral intermediates (INT1)}

The reaction mechanisms of $O$-acylhydroxamate with cysteine proteases have been carried out using high level $a b$ initio and DFT theories. The calculated frequencies show that tetrahedral intermediate (INT1) is in local minima on the potential energy surface (PES), and it is formed through the transition state (TS1). During the formation of tetrahedral intermediate (INT1), an intermolecular transition has occurred, in which, the protonated active cysteine molecule (hydrogen atom $\left(\mathrm{H}_{25}\right)$ of active thiol group of the 
Table 1. Selected optimized geometrical parameters (bond length $r$ in Å, bond angle A and torsional angle D in degrees), formation of product (P1) on reaction mechanism of $O$-acylhydroxamate with cysteine, calculated using DFT methods implementing 6-31 $+\mathrm{G}^{*}$ basis set.

\begin{tabular}{|c|c|c|c|c|c|c|c|c|c|c|}
\hline \multirow[b]{2}{*}{ Structures } & \multicolumn{2}{|r|}{$\mathrm{R}$} & \multicolumn{2}{|c|}{ TS1 } & \multicolumn{2}{|c|}{ INT1 } & \multicolumn{2}{|c|}{ TS2 } & \multicolumn{2}{|c|}{ P1 } \\
\hline & B3PW91 & B3LYP & B3PW91 & B3LYP & B3PW91 & B3LYP & B3LYP & B3PW91 & B3LYP & B3РW91 \\
\hline$r(1,2)$ & $1 \cdot 392$ & $1 \cdot 392$ & $1 \cdot 397$ & $1 \cdot 394$ & $1 \cdot 392$ & $1 \cdot 390$ & $1 \cdot 393$ & $1 \cdot 393$ & $1 \cdot 393$ & $1 \cdot 392$ \\
\hline$r(1,7)$ & & $1 \cdot 410$ & $1 \cdot 404$ & $1 \cdot 396$ & $1 \cdot 408$ & $1 \cdot 401$ & $1 \cdot 397$ & $1 \cdot 397$ & $1 \cdot 398$ & $1 \cdot 391$ \\
\hline$r(7,8)$ & .340 & $1 \cdot 340$ & $1 \cdot 342$ & $1 \cdot 338$ & $1 \cdot 346$ & $1 \cdot 343$ & $1 \cdot 358$ & & $1 \cdot 357$ & $1 \cdot 353$ \\
\hline$r(8,9)$ & $1 \cdot 369$ & $1 \cdot 369$ & $1 \cdot 369$ & $1 \cdot 364$ & $1 \cdot 368$ & $1 \cdot 363$ & $1 \cdot 325$ & $1 \cdot 326$ & $1 \cdot 325$ & $1 \cdot 320$ \\
\hline$r(8,10)$ & $1 \cdot 202$ & $1 \cdot 202$ & $1 \cdot 202$ & $1 \cdot 199$ & $1 \cdot 200$ & $1 \cdot 198$ & $1 \cdot 215$ & $1 \cdot 215$ & $1 \cdot 216$ & $1 \cdot 214$ \\
\hline$r(11,12)$ & $1 \cdot 399$ & $1 \cdot 399$ & $1 \cdot 468$ & $1 \cdot 462$ & $1 \cdot 465$ & 1.459 & $1 \cdot 372$ & $1 \cdot 372$ & $1 \cdot 372$ & $1 \cdot 368$ \\
\hline$r(12,13)$ & $1 \cdot 514$ & $1 \cdot 514$ & $1 \cdot 525$ & $1 \cdot 520$ & $1 \cdot 525$ & $1 \cdot 520$ & $1 \cdot 513$ & $1 \cdot 513$ & $1 \cdot 513$ & $1 \cdot 507$ \\
\hline$r(13,23)$ & 1.096 & 1.096 & & & & & & & & 1.094 \\
\hline$r(12,1$ & $1 \cdot 2$ & $1 \cdot 2$ & 99 & $1 \cdot 4$ & 09 & $1 \cdot 4$ & $1 \cdot 232$ & & $1 \cdot 233$ & $1 \cdot 230$ \\
\hline$r(12,24)$ & - & - & & & $1 \cdot 868$ & & - & - & - & - \\
\hline$r(24,26)$ & $1 \cdot 847$ & $1 \cdot 847$ & $1 \cdot 848$ & $1 \cdot 833$ & $1 \cdot 847$ & $1 \cdot 832$ & $1 \cdot 848$ & & $1 \cdot 848$ & $1 \cdot 844$ \\
\hline$r(24,11)$ & - & - & - & - & - & - & $1 \cdot 722$ & $1 \cdot 722$ & $1 \cdot 710$ & $1 \cdot 710$ \\
\hline$r(27,29)$ & $1 \cdot 526$ & 1.526 & $1 \cdot 5$ & 1.522 & 1.527 & 1.523 & & & $1 \cdot 528$ & $1 \cdot 524$ \\
\hline & & $1 \cdot 3$ & & $1 \cdot 3$ & & & & & $1 \cdot 349$ & $1 \cdot 343$ \\
\hline$r(29,31)$ & $1 \cdot 214$ & $1 \cdot 214$ & $1 \cdot 212$ & $1 \cdot 211$ & $1 \cdot 213$ & $1 \cdot 212$ & $1 \cdot 213$ & & $1 \cdot 213$ & $1 \cdot 211$ \\
\hline $\mathrm{A}(1,2,3)$ & $118 \cdot 499$ & $118 \cdot 513$ & $119 \cdot 405$ & $119 \cdot 456$ & $118 \cdot 223$ & $118 \cdot 891$ & $119 \cdot 212$ & 119 & $119 \cdot 213$ & 119 \\
\hline $\mathrm{A}(1,7,8)$ & $117 \cdot 613$ & $117 \cdot 374$ & $124 \cdot 085$ & $123 \cdot 988$ & $118 \cdot 900$ & $118 \cdot 633$ & $119 \cdot 6$ & $119 \cdot$ & $119 \cdot 774$ & $119 \cdot 629$ \\
\hline $\mathrm{A}(7,8,9)$ & $105 \cdot 127$ & $105 \cdot 027$ & $104 \cdot 136$ & $104 \cdot 057$ & $104 \cdot 678$ & $104 \cdot 584$ & $107 \cdot 892$ & $107 \cdot 892$ & $107 \cdot 986$ & 107.906 \\
\hline & & $128 \cdot 278$ & & $129 \cdot 1$ & $128 \cdot 144$ & $128 \cdot 185$ & $125 \cdot 448-$ & $125 \cdot 448$ & $125 \cdot 329$ & $125 \cdot 126$ \\
\hline $\mathrm{A}(9,11,12)$ & $115 \cdot 328$ & $115 \cdot 607$ & $107 \cdot 902$ & $107 \cdot 818$ & $108 \cdot 004$ & $107 \cdot 894$ & - & - & - & - \\
\hline $\mathrm{A}(11,12,13)$ & $121 \cdot 833$ & $121 \cdot 862$ & $113 \cdot 402$ & $113 \cdot 524$ & $113 \cdot 700$ & $113 \cdot 802$ & $121 \cdot 230$ & $115 \cdot 719$ & $116 \cdot 224$ & $115 \cdot 899$ \\
\hline $\mathrm{A}(11,12,14)$ & $113 \cdot 349$ & $113 \cdot 303$ & $107 \cdot 265$ & $107 \cdot 269$ & $107 \cdot 159$ & $107 \cdot 178$ & $115 \cdot 719$ & $121 \cdot 230$ & $127 \cdot 557$ & $121 \cdot 257$ \\
\hline $\mathrm{A}(26,27,2$ & $113 \cdot 461$ & $113 \cdot 4$ & & $113 \cdot 044$ & $113 \cdot 225$ & $113 \cdot 244$ & $113 \cdot 545$ & $113 \cdot 5$ & $113 \cdot 475$ & $109 \cdot 972$ \\
\hline $\mathrm{A}(24,26,27)$ & $114 \cdot 746$ & $114 \cdot 905$ & $111 \cdot 637$ & $111 \cdot 616$ & $111 \cdot 254$ & $111 \cdot 249$ & $111 \cdot 018$ & $111 \cdot 018$ & $111 \cdot 136$ & $111 \cdot 136$ \\
\hline $\mathrm{A}(28,27,29)$ & $106 \cdot 838$ & $106 \cdot 908$ & $106 \cdot 679$ & $106 \cdot 671$ & $106 \cdot 508$ & $106 \cdot 560$ & $106 \cdot 340$ & $106 \cdot 340$ & $106 \cdot 384$ & $106 \cdot 023$ \\
\hline $\mathrm{A}(30,29,31)$ & $122 \cdot 929$ & $123 \cdot 036$ & $123 \cdot 194$ & $123 \cdot 283$ & $123 \cdot 007$ & $123 \cdot 173$ & $123 \cdot 418$ & $123 \cdot 418$ & $123 \cdot 413$ & $123 \cdot 497$ \\
\hline $\mathrm{A}(24,12,13)$ & - & - & $112 \cdot 805$ & $112 \cdot 782$ & $112 \cdot 429$ & $112 \cdot 417$ & - & - & - & - \\
\hline $\mathrm{A}(26,24,11)$ & - & - & - & - & - & - & $100 \cdot 943$ & $100 \cdot 943$ & $100 \cdot 836$ & $100 \cdot 957$ \\
\hline $\mathrm{D}(24,26,27,28)$ & $-175 \cdot 947$ & $-176 \cdot 798$ & & & $-179 \cdot 904-$ & $-179 \cdot 863$ & $173 \cdot 349$ & $173 \cdot 447$ & $173 \cdot 349$ & $173 \cdot 777$ \\
\hline $\mathrm{D}(24,26,27,29)$ & $-64 \cdot 003$ & $-63 \cdot 328$ & $-63 \cdot 913$ & $-63 \cdot 566$ & $-60 \cdot 338$ & $-46 \cdot 782$ & $-67 \cdot 483$ & $-67 \cdot 371$ & $-67 \cdot 483$ & $-67 \cdot 254$ \\
\hline $\mathrm{D}(26,27,29,30)$ & $121 \cdot 528$ & $119 \cdot 978$ & $103 \cdot 491$ & $103 \cdot 029$ & $111 \cdot 823$ & $110 \cdot 526$ & $117 \cdot 541$ & $116 \cdot 712$ & $117 \cdot 541$ & $117 \cdot 691$ \\
\hline
\end{tabular}

cysteine molecule) is deprotonated to form a stable INT1. During the deprotonation, the hydrogen atom $\left(\mathrm{H}_{25}\right)$ of active thiol group is directed to the oxygen atom $\left(\mathrm{O}_{14}\right)$ and binds to acylhydroximate of peptide residue end. Likewise, similar process is found to occur in the enzymatic activity, where, the deprotonated cysteine enzyme $\mathrm{S}^{-}$ions bind to the stronger electrophile (positively charged) acylhydroximate chiral carbon atom $\mathrm{C}_{20}$ of the peptide residue end. Also, due to the proton transfer, $\mathrm{C}_{12}-\mathrm{O}_{14} \pi$ bond has been cleaved and a $\sigma$ bond is formed, the bond length between $\mathrm{C}_{12}-\mathrm{O}_{14}$ gets elongated and the elongation values are found to be $0 \cdot 193$ and $0.187 \AA$ at B3LYP and B3PW91/6-31 + G* levels of theory respectively, which has been confirmed not only by the elongation of the $\mathrm{C}_{12}-\mathrm{O}_{14}$ bond but also by the low electron density in AIM analysis. Moreover, bond between
$\mathrm{C}_{12}-\mathrm{S}_{24}$ atoms is not strong, so it can be easily cleaved and rearranged to form a stable product where the electron density and laplacian density of $\mathrm{C}_{12}-\mathrm{S}_{24}$ bond are found to be 0.169 and -0.253 a.u., which confirms the weak nature of the bond at TS1. The topological and AIM analyses of the hydrogen bonded network in the $O$-acylhydroxamate with cysteine proteases reaction mechanism have been shown in the tables 4 and 5. It is quite interesting to note that intermolecular weak hydrogen bonded network between the reactants $O$-acyl hydroxamates and protonated cysteine molecules are found between $\mathrm{N}_{28}$ $\mathrm{H}_{34} \ldots \mathrm{O}_{4}, \mathrm{O}_{26}-\mathrm{H}_{32} \ldots \mathrm{O}_{10}$ and $\mathrm{C}_{13}-\mathrm{H}_{23} \ldots \mathrm{S}_{24}$ atoms. Generally for hydrogen bonded complex, the electron density $(\rho)$ and laplacian of electron density $\left(\nabla^{2} \rho\right)$ are in the range of $0.002-0.34$ and $0.016-$ 0.139 a.u., respectively. ${ }^{19,20}$ In the present study, the 
Table 2. Selected optimized geometrical parameters (bond length $r$ in $\AA$, bond angle A and torsional angle $D$ in degrees), formation of product (P2) on reaction mechanism of $O$-acylhydroxamate with cysteine, calculated using DFT methods implementing 6-31 + G* basis set.

\begin{tabular}{|c|c|c|c|c|c|c|c|c|c|c|}
\hline \multirow[b]{2}{*}{ Structures } & \multicolumn{2}{|r|}{$\mathrm{R}$} & \multicolumn{2}{|r|}{ TS1 } & \multicolumn{2}{|c|}{ INT1 } & \multicolumn{2}{|r|}{ TS3 } & \multicolumn{2}{|r|}{ P1 } \\
\hline & B3LYP & B3PW91 & B3LYP & B3РW91 & B3LYP & P B3РW91 & B3LYP & B3PW91 & B3LYP & B3PW91 \\
\hline$r(1,2)$ & $1 \cdot 392$ & $1 \cdot 392$ & $1 \cdot 397$ & $1 \cdot 395$ & $1 \cdot 393$ & $1 \cdot 390$ & $1 \cdot 392$ & $1 \cdot 390$ & $1 \cdot 393$ & $1 \cdot 392$ \\
\hline$r(1,7)$ & $1 \cdot 410$ & $1 \cdot 410$ & $1 \cdot 403$ & $1 \cdot 396$ & $1 \cdot 408$ & 1.401 & 1.400 & $1 \cdot 393$ & $1 \cdot 391$ & $1 \cdot 391$ \\
\hline$r(7,8)$ & $1 \cdot 340$ & $1 \cdot 340$ & $1 \cdot 342$ & $1 \cdot 338$ & $1 \cdot 346$ & $1 \cdot 343$ & $1 \cdot 353$ & $1 \cdot 350$ & $1 \cdot 357$ & $1 \cdot 353$ \\
\hline$r(8,9)$ & $1 \cdot 369$ & $1 \cdot 369$ & $1 \cdot 369$ & $1 \cdot 364$ & $1 \cdot 368$ & $1 \cdot 363$ & $1 \cdot 220$ & $1 \cdot 218$ & $1 \cdot 325$ & $1 \cdot 320$ \\
\hline$r(8,10)$ & $1 \cdot 202$ & $1 \cdot 202$ & $1 \cdot 202$ & $1 \cdot 200$ & $1 \cdot 200$ & $1 \cdot 198$ & $1 \cdot 320$ & $1 \cdot 315$ & $1 \cdot 216$ & $1 \cdot 214$ \\
\hline$r(9,11)$ & 1.406 & 1.406 & $1 \cdot 442$ & 1.428 & 1.441 & 1.427 & - & - & - & - \\
\hline$r(11,12)$ & $1 \cdot 399$ & $1 \cdot 399$ & $1 \cdot 468$ & $1 \cdot 462$ & $1 \cdot 465$ & $1 \cdot 459$ & $1 \cdot 353$ & $1 \cdot 350$ & $1 \cdot 372$ & $1 \cdot 368$ \\
\hline$r(12,14)$ & $1 \cdot 216$ & $1 \cdot 216$ & $1 \cdot 409$ & 1.403 & 1.409 & 1.403 & $1 \cdot 239$ & $1 \cdot 237$ & $1 \cdot 233$ & $1 \cdot 230$ \\
\hline$r(24,26)$ & $1 \cdot 847$ & $1 \cdot 847$ & $1 \cdot 848$ & $1 \cdot 833$ & $1 \cdot 847$ & $1 \cdot 8$ & 1.852 & 1.836 & 1.833 & $1 \cdot 834$ \\
\hline$r(27,36)$ & 1.094 & 1.095 & 1.095 & 1.095 & 1.094 & 1.095 & $1 \cdot 091$ & 1.094 & $1 \cdot 091$ & $1 \cdot 092$ \\
\hline$r(26,27)$ & $1 \cdot 542$ & $1 \cdot 542$ & 1.550 & $1 \cdot 545$ & $1 \cdot 547$ & $1 \cdot 542$ & $1 \cdot 557$ & $1 \cdot 552$ & 1.545 & $1 \cdot 540$ \\
\hline$r(27,28)$ & $1 \cdot 464$ & $1 \cdot 464$ & $1 \cdot 463$ & $1 \cdot 456$ & $1 \cdot 464$ & $1 \cdot 457$ & $1 \cdot 454$ & $1 \cdot 447$ & $1 \cdot 464$ & $1 \cdot 457$ \\
\hline$r(29,30)$ & $1 \cdot 351$ & $1 \cdot 351$ & $1 \cdot 351$ & $1 \cdot 345$ & $1 \cdot 348$ & $1 \cdot 343$ & $1 \cdot 333$ & $1 \cdot 327$ & $1 \cdot 349$ & $1 \cdot 343$ \\
\hline$r(29,31)$ & $1 \cdot 214$ & $1 \cdot 213$ & $1 \cdot 212$ & $1 \cdot 211$ & $1 \cdot 214$ & $1 \cdot 21$ & $1 \cdot 219$ & $1 \cdot 217$ & $1 \cdot 213$ & $1 \cdot 211$ \\
\hline$r(12,24)$ & - & - & $1 \cdot 866$ & $1 \cdot 854$ & $1 \cdot 868$ & $1 \cdot 8$ & $1 \cdot 804$ & $1 \cdot 792$ & $1 \cdot 802$ & $1 \cdot 792$ \\
\hline$r(30,37)$ & 0.977 & 0.975 & 0.977 & 0.975 & $0 \cdot 977$ & 0.975 & 0.989 & 0.989 & 0.989 & 0.989 \\
\hline $\mathrm{A}(1,7,8)$ & $117 \cdot 613$ & $117 \cdot 374$ & $124 \cdot 085$ & $123 \cdot 988$ & $118 \cdot 900$ & $118 \cdot 633$ & $119 \cdot 147$ & $118 \cdot 798$ & $119 \cdot 217$ & $118 \cdot 941$ \\
\hline $\mathrm{A}(7,8,9)$ & $05 \cdot 127$ & $105 \cdot 027$ & $104 \cdot 136$ & $104 \cdot 057$ & $104 \cdot 678$ & $104 \cdot 584$ & $124 \cdot 874$ & $124 \cdot 757$ & $124 \cdot 897$ & $124 \cdot 799$ \\
\hline $\mathrm{A}(7,8,10)$ & $28 \cdot 172$ & $128 \cdot 278$ & $129 \cdot 008$ & $129 \cdot 147$ & $128 \cdot 144$ & $128 \cdot 185$ & $108 \cdot 483$ & $108 \cdot 456$ & $108 \cdot 475$ & $108 \cdot 430$ \\
\hline $\mathrm{A}(11,12,14)$ & $113 \cdot 349$ & $113 \cdot 303$ & $107 \cdot 265$ & $107 \cdot 269$ & $107 \cdot 159$ & $107 \cdot 178$ & $121 \cdot 271$ & $121 \cdot 298$ & $121 \cdot 892$ & $121 \cdot 910$ \\
\hline $\mathrm{A}(26,27,28)$ & $113 \cdot 461$ & $113 \cdot 455$ & $113 \cdot 054$ & $113 \cdot 044$ & $113 \cdot 225$ & $113 \cdot 244$ & 111.626 & 111.659 & 111.646 & $111 \cdot 703$ \\
\hline $\mathrm{A}(24,26,27)$ & $114 \cdot 040$ & $114 \cdot 905$ & $111 \cdot 637$ & $111 \cdot 616$ & $111 \cdot 254$ & $111 \cdot 249$ & $121 \cdot 563$ & $121 \cdot 698$ & $121 \cdot 519$ & $121 \cdot 622$ \\
\hline $\mathrm{A}(28,27,29)$ & $122 \cdot 929$ & $123 \cdot 036$ & $123 \cdot 194$ & $123 \cdot 283$ & $123 \cdot 007$ & $123 \cdot 173$ & $119 \cdot 938$ & $120 \cdot 038$ & $119 \cdot 925$ & $120 \cdot 038$ \\
\hline $\mathrm{A}(30,29,31)$ & - & - & $110 \cdot 393$ & $110 \cdot 249$ & $110 \cdot 420$ & $110 \cdot 245$ & $116 \cdot 108$ & $116 \cdot 005$ & $114 \cdot 768$ & $114 \cdot 684$ \\
\hline $\mathrm{A}(24,12,13)$ & - & - & $105 \cdot 967$ & $105 \cdot 933$ & 105.999 & $105 \cdot 997$ & $122 \cdot 532$ & $122 \cdot 609$ & $123 \cdot 290$ & $123 \cdot 357$ \\
\hline $\mathrm{A}(24,12,11)$ & - & - & $110 \cdot 393$ & $110 \cdot 249$ & $110 \cdot 420$ & $110 \cdot 245$ & $116 \cdot 108$ & $116 \cdot 005$ & $114 \cdot 768$ & $114 \cdot 684$ \\
\hline A $(24,12,14)$ & - & - & $105 \cdot 967$ & $105 \cdot 933$ & $105 \cdot 999$ & $105 \cdot 997$ & $122 \cdot 532$ & $122 \cdot 609$ & $123 \cdot 290$ & $123 \cdot 357$ \\
\hline $\mathrm{D}(12,24,26)$ & - & - & $102 \cdot 860$ & $102 \cdot 763$ & $102 \cdot 830$ & $102 \cdot 680$ & $103 \cdot 043$ & $102 \cdot 853$ & $103 \cdot 304$ & $103 \cdot 113$ \\
\hline $\mathrm{D}(11,12,24,26)$ & - & - & $-49 \cdot 575$ & $-50 \cdot 318$ & $-46 \cdot 782$ & $-46 \cdot 782$ & -157.569 & $-157 \cdot 550$ & $-158 \cdot 302$ & $-157 \cdot 943$ \\
\hline $\mathrm{D}(14,12,24,26)$ & - & - & $-172 \cdot 787$ & $-172 \cdot 787$ & $-170 \cdot 369-$ & $-170 \cdot 369$ & $25 \cdot 840$ & $25 \cdot 840$ & $24 \cdot 216$ & $24 \cdot 548$ \\
\hline $\mathrm{D}(24,26,27,29)$ & $-63 \cdot 328$ & - & $-63 \cdot 679$ & -63.566 & $-60 \cdot 679$ & $-60 \cdot 679$ & $-42 \cdot 993$ & $-42 \cdot 993$ & $-43 \cdot 376$ & $-42 \cdot 867$ \\
\hline $\mathrm{D}(24,26,27,28)$ & $176 \cdot 798$ & - & $177 \cdot 060$ & $177 \cdot 060$ & $179 \cdot 863$ & $179 \cdot 863$ & $-165 \cdot 979$ & $-165 \cdot 979$ & $-166 \cdot 306$ & $-165 \cdot 837$ \\
\hline
\end{tabular}

values of $\rho$ and $\nabla^{2} \rho$ are found to vary from 0.004 to 0.117 and $0.012-0.135 \mathrm{au}$, respectively. So the above values indicate that all the complexes are having intermolecular hydrogen bonds.

During the formation of tetrahedral intermediate INT1, the entire intermolecular hydrogen bonded networks $\mathrm{N}_{28}-\mathrm{H}_{34} \ldots \mathrm{O}_{11}, \mathrm{O}_{26}-\mathrm{H}_{32} \ldots \mathrm{O}_{10}$ and $\mathrm{C}_{13}-$ $\mathrm{H}_{23} \ldots \mathrm{S}_{24}$ of the reactants are cleaved in order to form a new intramolecular hydrogen bonded bridges between $\mathrm{C}_{2}-\mathrm{H}_{15} \ldots \mathrm{O}_{31}$ and $\mathrm{C}_{26}-\mathrm{H}_{3} \ldots \mathrm{O}_{10}$ atoms. As we know that hydrogen bonds are formed due to the charge transfer from the proton acceptor to proton donor, the amount of charge transfer is significant for the elongation and contraction of $\mathrm{X}-\mathrm{H}$ bond. For each donor and acceptor the stabilization energy $E$ is as- sociated with $i-j$ delocalization which is given by the following equation

$$
E^{(2)}=\Delta E_{i j}=q_{i} \frac{F^{2}(i, j)}{\varepsilon_{j}-\varepsilon_{i}} .
$$

where, $q_{i}$ the $i$ th donor orbital occupancy, $\varepsilon_{j}$, $\varepsilon_{i}$ are diagonal elements (orbital energies) and $F(i, j)$ is off diagonal elements respectively, associated with NBO Fock matrix.

Obviously, in INT1, the large stabilization energy of $2.07 \mathrm{kcal} / \mathrm{mol}$ for $\mathrm{C}_{2}-\mathrm{H}_{15} \ldots \mathrm{O}_{3}$ shows the maximum stability, when compared with the reactant hydrogen bonded network. But, the order of the molecular stability shows that the tetrahedral intermediate has 


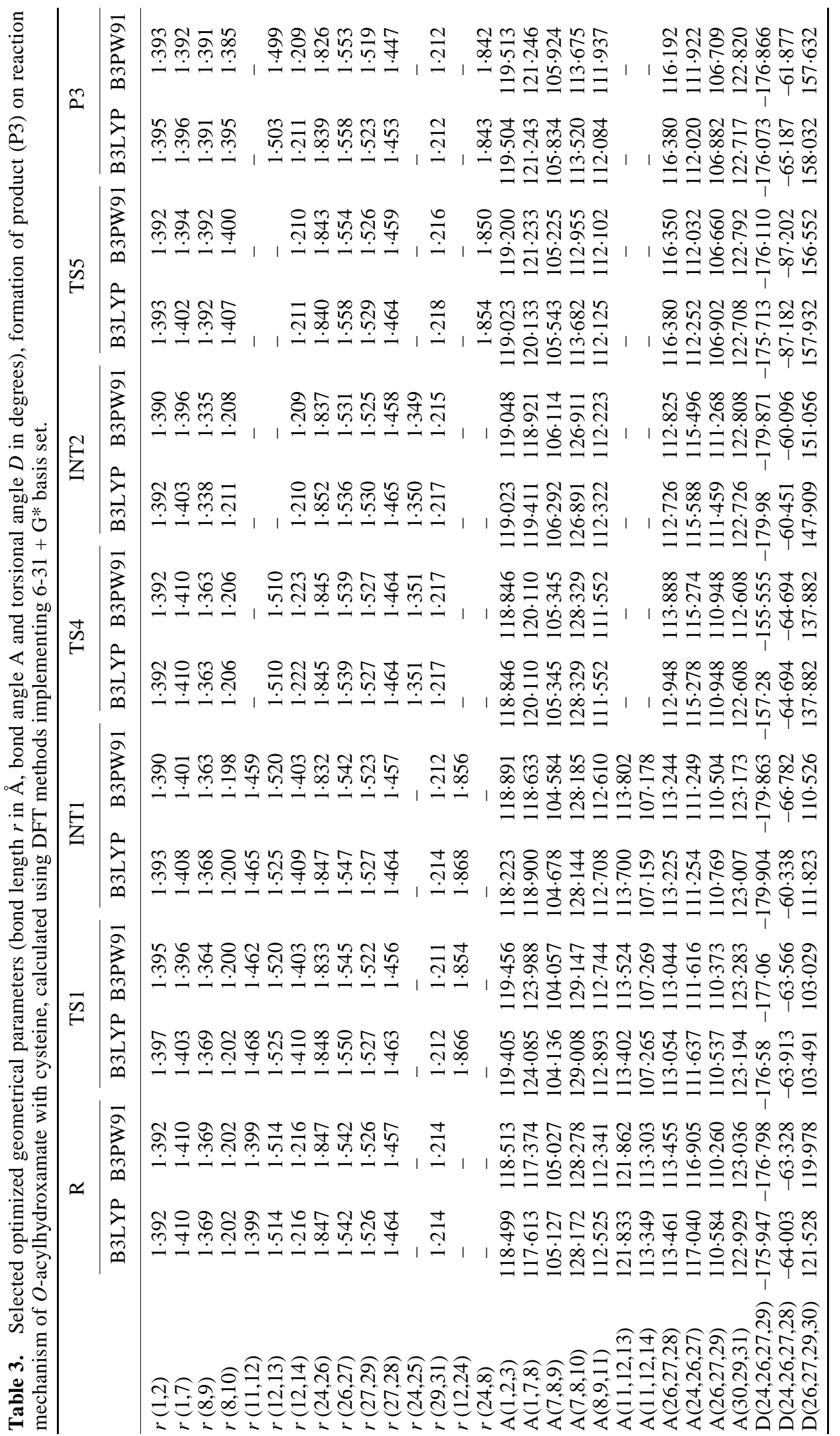


Table 4. Occupation number of the $\mathrm{X}-\mathrm{H}, \mathrm{Z}-\mathrm{X}$ bonds of the acceptor , the lone pairs of the acceptor atom and their corresponding energies (a.u), hydrogen bond length H...Y in $\AA$ and stabilization energy (in kcal/mol) of the interaction between lone pairs of acceptor atoms the anti-bonding orbitals are calculated using B3LYP theory at 6-31 + G* basis set.

\begin{tabular}{|c|c|c|c|c|c|c|c|c|}
\hline \multirow[b]{2}{*}{ System } & \multirow[b]{2}{*}{ Bonding } & \multicolumn{4}{|c|}{ Donor } & \multirow{2}{*}{$\begin{array}{l}\text { Acceptor } \\
n(\mathrm{Y})\end{array}$} & \multirow[b]{2}{*}{ H....Y } & \multirow{2}{*}{$\begin{array}{c}\mathrm{E}(2) n(y) \rightarrow \\
\sigma^{*}(\mathrm{X}-\mathrm{H})\end{array}$} \\
\hline & & $r(\mathrm{Z}-\mathrm{X})$ & $\sigma^{*}(\mathrm{Z}-\mathrm{X})$ & $r(\mathrm{X}-\mathrm{H})$ & $\sigma^{*}(\mathrm{X}-\mathrm{H})$ & & & \\
\hline $\mathrm{R}$ & $\begin{array}{c}\mathrm{N}_{28}-\mathrm{H}_{34} \ldots \mathrm{O}_{10} \\
\mathrm{~S}_{24}-\mathrm{C}_{26}-\mathrm{H}_{32} \ldots \mathrm{O}_{10} \\
\mathrm{C}_{12}-\mathrm{C}_{13}-\mathrm{H}_{23} \ldots \mathrm{S}_{24}\end{array}$ & $\begin{array}{l}- \\
1 \cdot 847 \\
1 \cdot 514\end{array}$ & $\begin{array}{l}- \\
0 \cdot 023 \\
0 \cdot 054\end{array}$ & $\begin{array}{l}1 \cdot 019 \\
1 \cdot 092 \\
1 \cdot 097\end{array}$ & $\begin{array}{l}0 \cdot 011 \\
0 \cdot 018 \\
0 \cdot 010\end{array}$ & $\begin{array}{l}1 \cdot 970 \\
1 \cdot 970 \\
1 \cdot 994\end{array}$ & $\begin{array}{l}2 \cdot 356 \\
2 \cdot 596 \\
3 \cdot 154\end{array}$ & $\begin{array}{l}1 \cdot 80 \\
0 \cdot 73 \\
1 \cdot 48\end{array}$ \\
\hline TS1 & $\begin{array}{c}\mathrm{S}_{24}-\mathrm{C}_{26}-\mathrm{H}_{33} \ldots \mathrm{O}_{10} \\
\mathrm{C}_{5}-\mathrm{C}_{6}-\mathrm{H}_{19} \ldots \mathrm{O}_{10}\end{array}$ & $\begin{array}{l}1 \cdot 848 \\
1 \cdot 310\end{array}$ & $\begin{array}{l}0 \cdot 028 \\
0 \cdot 015\end{array}$ & $\begin{array}{l}1 \cdot 092 \\
1 \cdot 086\end{array}$ & $\begin{array}{l}0 \cdot 023 \\
0 \cdot 015\end{array}$ & $\begin{array}{l}1 \cdot 971 \\
1 \cdot 813\end{array}$ & $\begin{array}{l}2 \cdot 435 \\
2 \cdot 254\end{array}$ & $\begin{array}{l}0 \cdot 84 \\
1 \cdot 38\end{array}$ \\
\hline INT1 & $\begin{array}{c}\mathrm{C}_{1}-\mathrm{C}_{2}-\mathrm{H}_{15} \ldots \mathrm{O}_{31} \\
\mathrm{~S}_{24}-\mathrm{C}_{26}-\mathrm{H}_{33} \ldots \mathrm{O}_{10}\end{array}$ & $\begin{array}{l}1 \cdot 393 \\
1 \cdot 847\end{array}$ & $\begin{array}{l}0 \cdot 026 \\
0 \cdot 028\end{array}$ & $\begin{array}{l}1 \cdot 085 \\
1 \cdot 092\end{array}$ & $\begin{array}{l}0 \cdot 016 \\
0 \cdot 022\end{array}$ & $\begin{array}{l}1 \cdot 973 \\
1 \cdot 972\end{array}$ & $\begin{array}{l}2 \cdot 396 \\
2 \cdot 462\end{array}$ & $\begin{array}{l}2 \cdot 07 \\
0 \cdot 74\end{array}$ \\
\hline TS3 & $\begin{array}{c}\mathrm{N}_{28}-\mathrm{C}_{27}-\mathrm{H}_{36} \ldots \mathrm{O}_{14} \\
\mathrm{O}_{30}-\mathrm{H}_{37} \ldots \mathrm{O}_{14}\end{array}$ & $\begin{array}{l}1 \cdot 454 \\
-\end{array}$ & $\begin{array}{l}0 \cdot 015 \\
-\end{array}$ & $\begin{array}{l}1 \cdot 091 \\
0 \cdot 989\end{array}$ & $\begin{array}{l}0 \cdot 019 \\
0 \cdot 046\end{array}$ & $\begin{array}{l}1 \cdot 958 \\
1 \cdot 958\end{array}$ & $\begin{array}{l}2 \cdot 421 \\
1 \cdot 815\end{array}$ & $\begin{array}{l}0 \cdot 51 \\
7 \cdot 44\end{array}$ \\
\hline P2 & $\begin{array}{c}\mathrm{N}_{28}-\mathrm{C}_{27}-\mathrm{H}_{36} \ldots \mathrm{O}_{14} \\
\mathrm{O}_{30}-\mathrm{H}_{37} \ldots \mathrm{O}_{14}\end{array}$ & $\begin{array}{l}1 \cdot 454 \\
-\end{array}$ & $\begin{array}{l}0 \cdot 015 \\
-\end{array}$ & $\begin{array}{l}1 \cdot 091 \\
0 \cdot 989\end{array}$ & $\begin{array}{l}0 \cdot 019 \\
0 \cdot 046\end{array}$ & $\begin{array}{l}1 \cdot 840 \\
1 \cdot 958\end{array}$ & $\begin{array}{l}2 \cdot 423 \\
1 \cdot 816\end{array}$ & $\begin{array}{l}0 \cdot 52 \\
7 \cdot 38\end{array}$ \\
\hline TS4 & $\mathrm{N}_{28}-\mathrm{H}_{35} \ldots \mathrm{O}_{10}$ & - & - & $1 \cdot 020$ & 0.013 & 1.969 & $2 \cdot 248$ & $3 \cdot 27$ \\
\hline INT2 & $\begin{array}{c}\mathrm{S}_{24}-\mathrm{C}_{6}-\mathrm{H}_{33} \ldots \mathrm{O}_{10} \\
\mathrm{~N}_{28}-\mathrm{H}_{35} \ldots \mathrm{O}_{10}\end{array}$ & $\begin{array}{l}1 \cdot 852 \\
-\end{array}$ & $\begin{array}{l}0 \cdot 025 \\
-\end{array}$ & $\begin{array}{l}1 \cdot 091 \\
1 \cdot 02\end{array}$ & $\begin{array}{l}0 \cdot 019 \\
0 \cdot 013\end{array}$ & $\begin{array}{l}1 \cdot 969 \\
1 \cdot 969\end{array}$ & $\begin{array}{l}2 \cdot 458 \\
2 \cdot 272\end{array}$ & $\begin{array}{l}1 \cdot 02 \\
2 \cdot 90\end{array}$ \\
\hline
\end{tabular}

Table 5. Topological analysis of electron density $(\rho)$, laplacian density $\left(\nabla^{2} \rho\right)$ and ellipticity $(\varepsilon)$ and hydrogen bond length R $(\AA)$ calculated using B3LYP method at 6-31 + G* basis set.

\begin{tabular}{|c|c|c|c|c|c|}
\hline Bonding & & $\mathrm{R}(\AA)$ & $\rho$ (a.u.) & $\nabla^{2} \rho$ (a.u.) & $\varepsilon$ (a.u.) \\
\hline $\mathrm{R}$ & $\begin{array}{c}\mathrm{N}_{28}-\mathrm{H}_{34} \ldots \mathrm{O}_{10} \\
\mathrm{~S}_{24}-\mathrm{C}_{26}-\mathrm{H}_{32} \ldots \mathrm{O}_{10} \\
\mathrm{C}_{12}-\mathrm{C}_{13}-\mathrm{H}_{23} \ldots \mathrm{S}_{24}\end{array}$ & $\begin{array}{l}2 \cdot 356 \\
2 \cdot 596 \\
3 \cdot 154\end{array}$ & $\begin{array}{l}0 \cdot 0097 \\
0 \cdot 0069 \\
0 \cdot 0050\end{array}$ & $\begin{array}{l}0 \cdot 0384 \\
0 \cdot 0261 \\
0 \cdot 0147\end{array}$ & $\begin{array}{l}0 \cdot 0692 \\
0 \cdot 0515 \\
0 \cdot 0445\end{array}$ \\
\hline TS1 & $\begin{array}{c}\mathrm{S}_{24}-\mathrm{C}_{26}-\mathrm{H}_{33} \ldots \mathrm{O}_{10} \\
\mathrm{C}_{5}-\mathrm{C}_{6}-\mathrm{H}_{19} \ldots \mathrm{O}_{10}\end{array}$ & $\begin{array}{l}2 \cdot 435 \\
2 \cdot 254\end{array}$ & $\begin{array}{l}0 \cdot 0090 \\
0 \cdot 0174\end{array}$ & $\begin{array}{l}0 \cdot 0335 \\
0 \cdot 0658\end{array}$ & $\begin{array}{l}0 \cdot 0600 \\
0 \cdot 233\end{array}$ \\
\hline INT1 & $\begin{array}{c}\mathrm{C}_{1}-\mathrm{C}_{2}-\mathrm{H}_{15} \ldots \mathrm{O}_{31} \\
\mathrm{~S}_{24}-\mathrm{C}_{26}-\mathrm{H}_{33} \ldots \mathrm{O}_{10}\end{array}$ & $\begin{array}{l}2 \cdot 396 \\
2 \cdot 462\end{array}$ & $\begin{array}{l}0 \cdot 0091 \\
0 \cdot 0091\end{array}$ & $\begin{array}{l}0 \cdot 0348 \\
0 \cdot 0328\end{array}$ & $\begin{array}{l}0 \cdot 0718 \\
0 \cdot 0660\end{array}$ \\
\hline TS3 & $\begin{array}{c}\mathrm{N}_{28}-\mathrm{C}_{27}-\mathrm{H}_{36} \ldots \mathrm{O}_{14} \\
\mathrm{O}_{30}-\mathrm{H}_{37} \ldots \mathrm{O}_{14}\end{array}$ & $\begin{array}{l}2 \cdot 421 \\
1 \cdot 815\end{array}$ & $\begin{array}{l}0 \cdot 0130 \\
0 \cdot 0347\end{array}$ & $\begin{array}{l}0 \cdot 0478 \\
0 \cdot 1107\end{array}$ & $\begin{array}{l}0 \cdot 2125 \\
0 \cdot 0334\end{array}$ \\
\hline P2 & $\begin{array}{c}\mathrm{N}_{28}-\mathrm{C}_{27}-\mathrm{H}_{36} \ldots \mathrm{O}_{14} \\
\mathrm{O}_{30}-\mathrm{H}_{37} \ldots \mathrm{O}_{14}\end{array}$ & $\begin{array}{l}2 \cdot 423 \\
1 \cdot 816\end{array}$ & $\begin{array}{l}0.0130 \\
0.0346\end{array}$ & $\begin{array}{l}0 \cdot 0475 \\
0 \cdot 1317\end{array}$ & $\begin{array}{l}0 \cdot 2055 \\
0 \cdot 0102\end{array}$ \\
\hline TS4 & $\mathrm{N}_{28}-\mathrm{H}_{35} \ldots \mathrm{O}_{10}$ & $2 \cdot 248$ & 0.0118 & 0.0466 & $0 \cdot 0673$ \\
\hline INT2 & $\begin{array}{c}\mathrm{S}_{24}-\mathrm{C}_{6}-\mathrm{H}_{33} \ldots \mathrm{O}_{10} \\
\mathrm{~N}_{28}-\mathrm{H}_{35} \ldots \mathrm{O}_{10}\end{array}$ & $\begin{array}{l}1 \cdot 969 \\
1 \cdot 969\end{array}$ & $\begin{array}{l}0.0092 \\
0 \cdot 0115\end{array}$ & $\begin{array}{l}0 \cdot 0335 \\
0 \cdot 0445\end{array}$ & $\begin{array}{l}0 \cdot 0279 \\
0 \cdot 0570\end{array}$ \\
\hline
\end{tabular}

less stability than the reactant. This may be due to cleavage of large number of hydrogen bonded networks in INT1. At TS1, the bond lengths $\mathrm{N}_{11}-\mathrm{C}_{12}$, $\mathrm{C}_{12}-\mathrm{C}_{13}, \mathrm{C}_{12}-\mathrm{O}_{14}$ gets elongated and the elongation values are found to be $0 \cdot 068,0.010$ and $0.193 \AA$ respectively.

In TS1, the intramolecular hydrogen bonded networks $\mathrm{C}_{26}-\mathrm{H}_{3} \ldots \mathrm{O}_{10}, \mathrm{C}_{6}-\mathrm{H}_{19} \ldots \mathrm{O}_{10}$ have been observed. The stabilization energies of $\mathrm{C}_{26}-\mathrm{H}_{3} \ldots \mathrm{O}_{10}$ and $\mathrm{C}_{6}-$ $\mathrm{H}_{19} \ldots \mathrm{O}_{10}$ are found to be 0.84 and $1.38 \mathrm{kcal} / \mathrm{mol}$ re- spectively, but this hydrogen bonded bridge has been cleaved during the formation of tetrahedral intermediate INT1. The (IRC) calculations connect the transition state between the reactant and tetrahedral intermediate INT1. The energy barrier between the reactant to transition state TS1 is found be $15 \cdot 24$, 12.05 and $4.07 \mathrm{kcal} / \mathrm{mol}$ at B3LYP/6-31 $+\mathrm{G}^{*}$, B3PW91/6-31 + G* and MP2//B3LYP/6-31 + G* levels of theory, respectively. During the formation of the tetrahedral intermediate INT1, $\mathrm{N}_{11}-\mathrm{C}_{12}$ and 

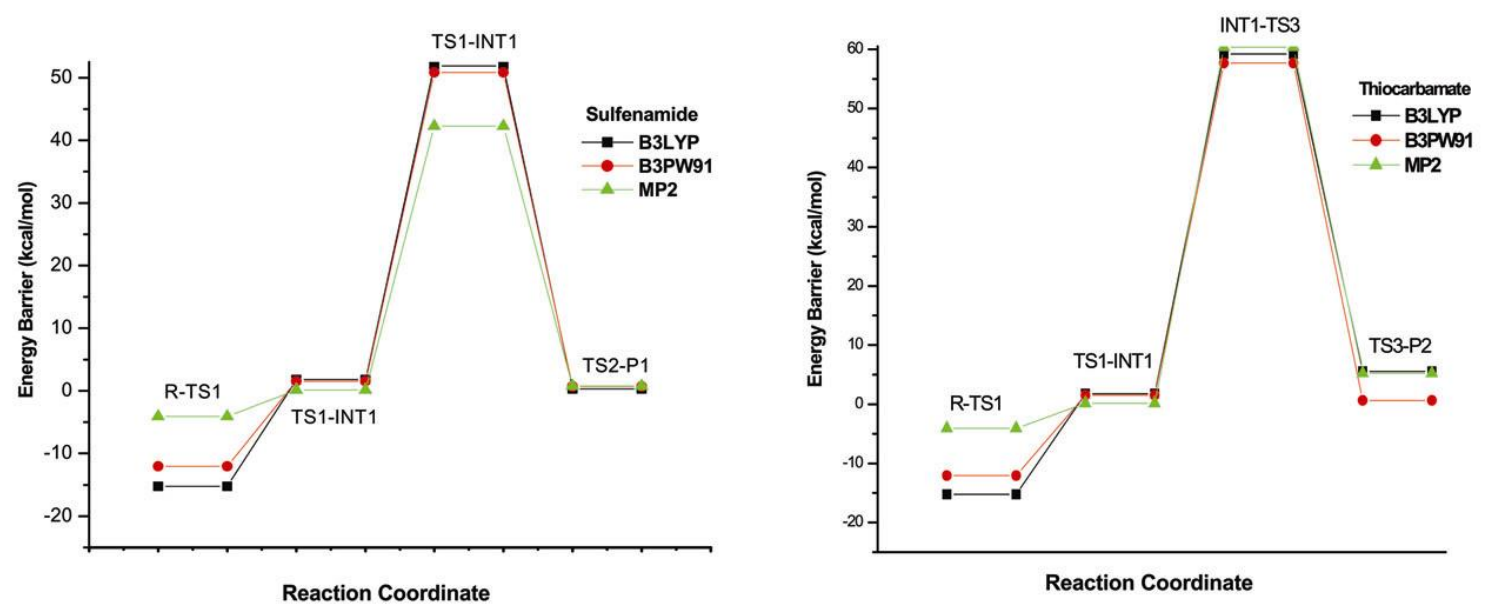

Figure 2. Calculated energy barrier $(\mathrm{kcal} / \mathrm{mol})$ for the formation of products $\mathrm{P} 1$ and P2 DFT and $a b$ initio methods implementing 6-31 $+\mathrm{G}^{*}$ basis set.

$\mathrm{C}_{12}-\mathrm{O}_{14}$ bonds get shortened and their respective values are given in table 1 . The energy barrier between TS1 and INT1 is found to be $1.79,1.53$ and $0 \cdot 12 \mathrm{kcal} / \mathrm{mol}$ at B3LYP/6-31 + G*, B3PW91/6$31+\mathrm{G}^{*}$ and MP2//B3LYP/6-31 + G* levels of theory respectively. In spite of the structural resemblance between TS1 and INT1, the internal geometry shows some small discrepancies. The INT1 structure is found to be less stable than the reactant which is indicated by the reaction barrier of -13.45 and $-10 \cdot 51 \mathrm{kcal} / \mathrm{mol}$ calculated at B3LYP/6-31 + G* and B3PW91/6-31 + G* levels of theory.

\subsection{Breakdown of the tetrahedral intermediate} (INT1)

The stable tetrahedral intermediate INT1 is a bridge between the reactant and product on the cysteine proteases inhibitor reaction profile. The previous calculations by Perakyla et $a l^{21}$ were based on the assumption that the transition state structures during the formation/breakdown of the tetrahedral intermediate are very similar to that of the tetrahedral intermediate. ${ }^{21-24}$ In the present calculation, the transition state structures TS1, TS2, TS3, and TS4 are found to be similar to that of the INT1, INT2, P1, P2, P3 and reactants. Additionally during the breakdown of the stable tetrahedral intermediate INT1, two stable by-products sulfenamide and thiocarbamate have been formed through TS2 and TS3 on the reaction profile of the cysteine proteases reaction mechanisms.

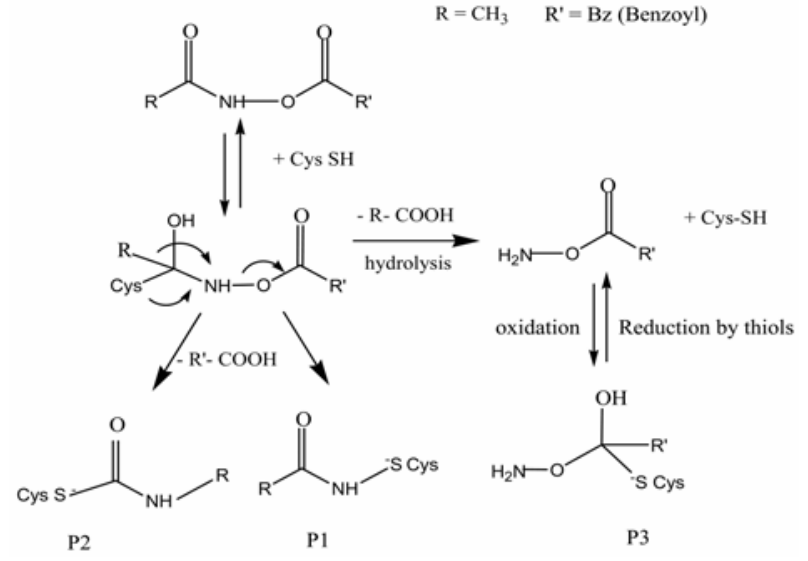

Scheme 1. Proposed mechanisms of inhibition of cysteine proteases by O-acylhydroxamate.

3.2a Formation of sulfenamide (P1): It is also one of the by-products and very strong inhibitor of cysteine proteases reaction mechanism profile, where the sulfenamide $\mathrm{P} 1$ is formed through the transition state TS2, and again the bond between $\mathrm{O}_{14}-\mathrm{H}_{25}$ atoms in the tetrahedral intermediate INT1 is cleaved, therefore, the $\sigma$ bond between $\mathrm{O}_{14}-\mathrm{C}_{12}$ atoms changes to the $\pi$ bond character. In the mean time, enzymatic cysteine proteases ions are cleaved from the acylhydroximate chiral carbon atom $\mathrm{C}_{12}$ and strongly bind to the strong electrophile $\mathrm{N}_{11}$ nitrogen atom of the $O$-acylhydroxamate. Due to the electrophilic attack of cysteine ions, bond between $\mathrm{N}_{11}-\mathrm{O}_{9}$ atoms is cleaved. During the formation of TS2, the bond length of $\mathrm{S}_{24}-\mathrm{C}_{26}$ atoms gets elongated by $0.015 \AA$ at 
B3PW91/6-31 + G* level of theory. At the same time, the bond lengths $\mathrm{C}_{8}-\mathrm{N}_{11}, \mathrm{~N}_{11}-\mathrm{C}_{12}$ and $\mathrm{C}_{12}-\mathrm{O}_{14}$ are shortened by $0.168,0.086$ and $0.170 \AA$ at B3LYP/6-31 + G* level of theory respectively. The IRC calculation connects the transition state between the tetrahedral intermediate INT1 and the product $\mathrm{P} 1$. The energy barrier for formation of the transition state structure TS2 is calculated to be 51.86 , $\quad 50.81$ and $42.23 \mathrm{kcal} / \mathrm{mol}$ at B3LYP/6$31+\mathrm{G}^{*}$, B3PW91/6-31 + G*, and MP2//B3LYP/6$31+\mathrm{G}^{*}$, levels of theory respectively and also, during the formation of TS2, the entire intramolecular hydrogen bonds network have been cleaved. The energy barrier on each steps of the reaction profile of the thiocarbamate $\mathrm{P} 1$ is calculated and the results are presented in figure 1 . The energy barrier between the TS2 and product $\mathrm{P} 1$ is found to be $0.32,0.73$ and $0.75 \mathrm{kcal} / \mathrm{mol}$ at B3LYP/6-31 + G*, B3PW91/6$31+\mathrm{G}^{*}$ and MP2//B3LYP $6-31+\mathrm{G}^{*}$ levels of theory respectively. Due to the occurrence of low energy barrier between TS2 and the product P1, the TS2 was easily re-arranged to form a stable product $\mathrm{P} 1$. Also, the product $\mathrm{P} 1$ was more stable than the reactant, and the reaction energy was found to be 38.73 , 39.57 and $39.53 \mathrm{kcal} / \mathrm{mol}$ at B3LYP/6-31 + G*, B3PW91/6-31 + G* and MP2//B3LYP/6-31 + G* levels of theory respectively. Additionally, the negative values of the reaction energies indicate that, the overall reaction profile of $\mathrm{P} 1$ is found to be exothermic.

During the formation of sulfenamide P1, the bond length between $S_{24}-N_{11}$ atoms is shortened by 0.115 and $0 \cdot 011 \AA$ at B3LYP/6-31 + G* and B3PW91/6$31+\mathrm{G}^{*}$ levels of theory when compared with TS2 and INT1 states. The stability of the $\mathrm{S}_{24}-\mathrm{N}_{11}$ bond has been calculated by electron density and laplacian density using AIM analysis. The electron density and laplacian density of $\mathrm{S}_{24}-\mathrm{N}_{11}$ bond are found to be $0 \cdot 186$, and $-0 \cdot 167$ a.u. at B3LYP/6-31 + G* level of theory, which shows a strong covalent nature of the bonding. This result confirms the inhibiting nature of the product $\mathrm{P} 1$.

3.2b Formation of thiocarbamate P2: On the irreversible reaction mechanism of cysteine proteases with $O$-acylhydroxamate, thiocarbamate $\mathrm{P} 2$ is also one of the byproduct. The calculated frequency for thiocarbamate P2 at the above mentioned levels of theory confirmed that the structures are on real local minima on the potential energy surface (PES), whereas, the product $\mathrm{P} 2$ is formed through the tran- sition state TS3. The numerical frequency calculation shows that TS3 has one imaginary frequency. During the formation of transition state TS3, the entire intra-molecular hydrogen bonded networks in the tetrahedral intermediate INT1 have been cleaved, to form new hydrogen bonded networks $\mathrm{C}_{27}-\mathrm{H}_{36} \ldots$ $\mathrm{O}_{14}$ and $\mathrm{O}_{30}-\mathrm{H}_{37} \ldots \mathrm{O}_{14}$ in TS3, where the stabilization energies of the $\mathrm{C}_{27}-\mathrm{H}_{36} \ldots \mathrm{O}_{14}$ and $\mathrm{O}_{30}-\mathrm{H}_{37} \ldots \mathrm{O}_{14}$ are found to be 7.44 and $0.51 \mathrm{kcal} / \mathrm{mol}$ at B3LYP/6$31+G^{*}$ level of theory. The IRC calculations connect the transition state between the tetrahedral intermediate INT1 and the product P2. The energy barrier on each steps of the reaction profile of the sulfenamide $\mathrm{P} 2$ is calculated and the results are presented in figure 2 . The energy barrier between the tetrahedral intermediate INT1 and transition state TS3 is found to be $59 \cdot 20,57 \cdot 65$ and $60 \cdot 36 \mathrm{kcal} / \mathrm{mol}$ at B3LYP/6-31 + G*, B3PW91/6-31+ G*1 and MP2//B3LYP/6-31 + G* levels of theory. In TS3, the bond lengths of $\mathrm{C}_{12}-\mathrm{O}_{14}, \mathrm{C}_{12}-\mathrm{S}_{24}$ and $\mathrm{N}_{11}-\mathrm{C}_{12}$ get contracted by $0 \cdot 170,0 \cdot 064$ and $0.111 \AA$ respectively at B3LYP/6-31 + G* level of theory. Obviously at the same time, bond length of $\mathrm{C}_{26}-\mathrm{C}_{27}$ gets elongated by 0.016 and $0.015 \AA$ at B3LYP/6-31 + G* and B3PW91/6-31 + G* levels of theory. The energy barrier between TS3 and product P2 is found to be 5.52, 0.65 and $5.25 \mathrm{kcal} / \mathrm{mol}$ at the above levels of theory. Also, during the formation of product P2, bond between deprotonated cysteine ions and $O$ acylhydroxamate, $\mathrm{N}_{12}-\mathrm{S}_{24}$ further contracts to form a strong covalent bond. In addition to that, the electron density between $\mathrm{N}_{12}-\mathrm{S}_{24}$ atoms also confirms the strong covalent nature. The product $\mathrm{P} 2$ is more stable than the reactant and the energy barrier between the reactant and product $\mathrm{P} 2$ is found to be 51.26, 47.75 and $45.98 \mathrm{kcal} / \mathrm{mol}$ at B3LYP/6-31 + G*, B3PW91/6-31 + G* and MP2//B3LYP/6-31 + G* levels of theory respectively. Owing to the occurrence of the heavy energy barrier, the formation of product $\mathrm{P} 2$ is less favoured than product $\mathrm{P} 1$ on this reaction profile. The product $\mathrm{P} 2$ is found to be more stable than the reactant $\mathrm{R}$; hence, its overall reaction profile of $\mathrm{P} 2$ is found to be exothermic nature.

\subsection{Formation and breakdown of INT2}

The breakdown of the tetrahedral intermediate INT1 into two stable products P1 and P2 have been formed on the acid hydrolysis of the irreversible process. The base hydrolysis process of the reversible reaction mechanism on the cysteine proteases inhibitors, 
results in the formation of a stable intermediate INT2, which has been formed through the transition state TS4. The numerical frequency calculation has also confirmed this TS4 state. During the formation of the TS4, the bond length of $\mathrm{C}_{1}-\mathrm{O}_{7}, \mathrm{C}_{8}-\mathrm{O}_{10}$ and $\mathrm{C}_{27}-\mathrm{N}_{28}$ gets elongated and the values are found to be 0.010 , 0.007 and $0.006 \AA$ at B3PW91/6-31 + G* level of the theory respectively. At the same time, the bond between $\mathrm{C}_{12}-\mathrm{C}_{13}, \mathrm{C}_{12}-\mathrm{O}_{14}$ and $\mathrm{C}_{26}-\mathrm{C}_{27}$ atoms are shortened whose values are found to be $0 \cdot 015,0 \cdot 186$ and $0.007 \AA$ at B3LYP/6-31 + G* level of theory respectively. During the formation of transition state TS4, the bond between $\mathrm{O}_{14}-\mathrm{H}_{25}$ atoms at INT1 gets cleaved on hydrolysis, meanwhile an electron shift occurred between $\mathrm{O}_{14}-\mathrm{H}_{25}$, and $\mathrm{O}_{14}-\mathrm{C}_{12}$ bonds. Due to this electron shift, the $\sigma$ bond character between $\mathrm{C}_{12}-\mathrm{O}_{14}$ atoms once again changed to a $\pi$ bond character. The bond length between $\mathrm{C}_{12}-\mathrm{O}_{14}$ atoms gets contract and the values are found to be 0.199 and 0.191 $\AA$ at B3LYP and B3PW91/6-31 + G* levels of theory. At the same time, the loosely bonded $\mathrm{S}_{24}-\mathrm{C}_{14}$ atoms gets cleaved; once again the loosely bonded cysteine enzyme $\mathrm{S}^{-}$ions get separated from acylhydroximate carbon atom $\mathrm{C}_{14}$ and bind with $\mathrm{H}^{+}$ion. The energy barrier between the INT1 and TS4 is found to be $76.44,76.40$ and $76.23 \mathrm{kcal} / \mathrm{mol}$ at the above levels of theory. The occurrences of the high energy barrier between INT1 and TS4 states might be due to the hydrolysis with water molecule.

The entire intramolecular hydrogen bonded networks $\mathrm{C}_{2}-\mathrm{H}_{5} \ldots \mathrm{O}_{31}$ and $\mathrm{C}_{26}-\mathrm{H}_{3} \ldots \mathrm{O}_{10}$ of INT1 are cleaved in order to form a new intermolecular hydrogen bond on $\mathrm{N}_{28}-\mathrm{H}_{35} \ldots \mathrm{O}_{10}$ atoms at TS4 and the stabilization energy of $\mathrm{N}_{28}-\mathrm{H}_{35} \ldots \mathrm{O}_{10}$ is found to be $3.27 \mathrm{kcal} / \mathrm{mol}$ at B3LYP $/ 6-31+\mathrm{G}^{*}$ level of theory. During the formation of INT2, the bond length $\mathrm{C}_{1}-\mathrm{O}_{7}$,

Table 6. Calculated energy barrier $(\mathrm{kcal} / \mathrm{mol})$ for the reaction mechanism of cystein with $O$-acyl hydroxamates at DFT and $a b$ initio methods implementing $6-31+\mathrm{G}^{*}$ basis set.

\begin{tabular}{lrrr}
\hline System & B3LYP & B3PW91 & MP2 \\
\hline R-TS1 & $-15 \cdot 24$ & $-12 \cdot 05$ & $-4 \cdot 07$ \\
TS1-INT1 & $1 \cdot 79$ & $1 \cdot 53$ & $0 \cdot 12$ \\
INT1-TS2 & $51 \cdot 86$ & $50 \cdot 81$ & $42 \cdot 23$ \\
TS2-P1 & $0 \cdot 32$ & $0 \cdot 73$ & $0 \cdot 75$ \\
INT1-TS3 & $59 \cdot 20$ & $57 \cdot 65$ & $60 \cdot 36$ \\
TS3-P2 & $5 \cdot 52$ & $0 \cdot 65$ & $5 \cdot 25$ \\
TS4-INT2 & $6 \cdot 23$ & $6 \cdot 70$ & $6 \cdot 46$ \\
INT2-TS5 & $20 \cdot 56$ & $20 \cdot 63$ & $18 \cdot 23$ \\
TS5-P3 & $8 \cdot 92$ & $10 \cdot 26$ & $7 \cdot 43$ \\
\hline
\end{tabular}

$\mathrm{C}_{12}-\mathrm{O}_{14}$ and $\mathrm{S}_{24}-\mathrm{H}_{25}$ get contracted and the contracted values are found to be $0.014,0.014$ and $0.002 \AA$ at the B3PW91/6-31+G* level of theory. The intermolecular hydrogen bonds $\mathrm{N}_{28}-\mathrm{H}_{35} \ldots \mathrm{O}_{10}$ and $\mathrm{C}_{26}-\mathrm{H}_{33} \ldots \mathrm{O}_{10}$ are observed at INT2, and the stabilization energies are found to be 2.90 and $1.02 \mathrm{kcal} / \mathrm{mol}$ at B3LYP/ $/ 6-31+\mathrm{G}^{*}$ level of theory. The energy barrier between TS4 and INT2 is found to be $6 \cdot 26,6.704$ and $6.46 \mathrm{kcal} / \mathrm{mol}$ at B3LYP/631+G*, B3PW91/6-31+G* and MP2//B3LYP/6$31+G^{*}$ levels of theory respectively.

3.3a Formation of P3: During the oxidation of the intermediate INT2, the stable intermediate INT2 structure breaks down to form a product P3 through the transition state TS5. The numerical frequency calculation has confirmed this TS5 state. During the formation of product P3, an intermolecular transition has been occurred, in which the cysteine molecule, the $\mathrm{S}-\mathrm{H}$ bond is once again deprotonated to form the stable product P3. While on the deprotonation, hydrogen atom $\mathrm{H}_{25}$ of the cysteine molecule was directed to the oxygen atom $\mathrm{O}_{10}$ and binds strongly with $O$-acylhydroxamate molecule. At the same time, the deprotonated enzymatic cysteine $\mathrm{S}^{-}$ ions bind once again to strong electrophile of $O$ acylhydroxamate carbon atom $\mathrm{C}_{8}$. Meanwhile, the $\pi$ bond character between $\mathrm{O}_{10}-\mathrm{C}_{8}$ atoms has been changed to $\sigma$ bond character. The bond length between $\mathrm{O}_{10}-\mathrm{C}_{8}$ gets elongated and the values are found to be $0 \cdot 187$ and $0.177 \AA$ at B3LYP/6-31 + G* and B3PW91/6$31+G^{*}$ levels of theory. The changes of the bonds are confirmed by the structural parameters (elongation of bond length) and topological analysis (low electron density at bond critical point). The energy barrier between INT2 and transition state TS5 is found to be $13 \cdot 56,20 \cdot 63$, and $10.23 \mathrm{kcal} / \mathrm{mol}$ at the above levels of theory respectively. During the oxidation of INT2, the entire intra-molecular and inter-molecular hydrogen bonds break down to form the stable product P3, also, the stability of the product P3 is low on comparing with the intermediate INT2. The variation of the stability might be due to the breakdown of all intra-molecular and inter-molecular hydrogen bonded networks. The energy barrier between INT2 and the product P3 is found to be $20.48,30.89$ and $19.40 \mathrm{kcal} / \mathrm{mol}$ at B3LYP/6-31 + G*, B3PW91/6$31+\mathrm{G}^{*}$ and MP2//B3LYP/6-31 + G* levels of theory respectively. At product $\mathrm{P} 3$, the bond between cysteine and $\mathrm{O}$-acylhydroxamate $\mathrm{C}_{8}-\mathrm{S}_{24}$ atoms is strong which shows the inhibiting nature of the 
product P3. The energy barrier between the TS5 and P3 is found to be $6.92,10.26$ and $9.17 \mathrm{kcal} / \mathrm{mol}$ at B3LYP/6-31 + G*, B3PW91/6-31 + G* and MP2// B3LYP/6-31 + G* levels of theory respectively. The active enzymatic cysteine is completely changed to inactive form at product P3. The products P1, P2 and P3 are energetically more stable than the reactant and hence the reaction enthalpy is found to be exothermic. The product P3, when reduced by thiol, yields the intermediate INT2 once again, as a result of the reversible process.

\section{Conclusion}

In this article, we have studied the reaction mechanism of the cysteine proteases with $O$-acylhydroxamate using B3LYP/6-31 + G* and B3PW91/6-31 + G* levels of density functional theory and MP2//B3LYP/ $6-31+\mathrm{G}^{*}$ level of $a b$ initio theory in gas phase. After the breakdown of tetrahedral intermediate INT1, small 1-2 aniontropic has formed and re-arranged to give stable by-products sulfenamide P1 and thiocarbamate P2 with considerable energy loss. The energy barrier between the tetrahedral intermediate INT1 and product P1 is found be low when compared to that of the product P2, so it favours to the formation of the reaction steps resulting $\mathrm{P} 1$ and this result agrees with experimental process. ${ }^{7}$ The strong binding nature of $\mathrm{C}_{12}-\mathrm{S}_{24}, \mathrm{~N}_{11}-\mathrm{S}_{24}$ and $\mathrm{C}_{8}-\mathrm{S}_{24}$ bonds with P1, P2 and P3 of the reaction step shows the inhibiting nature of the $O$-acylhydroxamate with cysteine proteases. AIM analyses agree with the results. This theoretical calculation shows that, the tetrahedral intermediate structure is also a stable species (not TS structure) though it was not detected by any experimental techniques. The IRC calculations connect the transition state between R-INT1, INT1-P1 and INT1-P2. The reaction energy is found to be exothermic in the formation of product P1, P2 and P3. The stabilization energy of intra-molecular and inter-molecular hydrogen bonds in the reaction steps of cysteine with $O$-acylhydroxamate has been calculated using AIM and NBO analyses.

\section{Acknowledgments}

The authors are thankful to the Department of Science and Technology, Government of India for the financial assistance to create the Central Computer Lab under the DST-FIST Programme.

\section{References}

1. (a) Fersht A 1999 Structure and mechanism in protein science. A guide to enzyme catalysis and protein folding (New York: W.H. Freeman and Company) 2nd edn; (b) Jencks W P 1987 Catalysis in chemistry and enzymology (New York: Dover Publications)

2. Walkinshaw M D 1992 Med. Res. Rev. 12317

3. Dermuth H-U 1990 J. Enzyme Inhib. 3249

4. Hans-Ulrich Demuth, Angelika Schierhorn, Philip Bryan, Ralph Hofke, Heidrrum Kirschke and Dieter Bromme 1996 Biochim. Biophys. Acta 1295179

5. Rawlings N D and Barrett A J 1999 Nucl. Acids Res. 27325

6. McGrath M E 1999 Ann. Rev. Biophys. Biomol Struct. 28181

7. Hans-Hartwing Otto and Tanja Schirmeister 1997 Chem. Rev. 97133

8. Valerie J, Robinson J, Coles, Smith R A and Allen Krantz 1991 J. Am. Chem. Soc. 1137760

9. (a) Fischer G, Demuth H-U and Barth A 1983 Pharmazie 38 249; (b) Demuth H-U, Neumann U, Schaper C, Fischer G and Barth A 1988 J. Enzyme Inhib. 2 129

10. Bromme D, Fittkau S and Demuth H-U 1989 J. Biochem. 263861

11. Smith R A, Peter J, Coles, Spencer R W, Copp L J, Jones C S and Krantz, A 1988 Biochem. Biophys. Res. Commun. 1551201

12. Groutas W C, Stanga M A and Brubaker M J 1989 J. Am. Chem. Soc. 1111931

13. Marcin Hoffmann, Jacek Rrychlewski, Maria Chrzanowska and Tadeusz Hermann $2001 \mathrm{~J}$. Am. Chem. Soc. 1236404

14. (a) Fuki K 1970 J. Phys. Chem. 74 4161; (b) Ishida K, Morokuma K and Komornicki A 1977 J. Chem. Phys. 662153

15. Cioslowski J 1994 Chem. Phys. Lett. 151

16. Carpenter J E and Weinhold F 1988 J. Mol. Struct. (Theochem) 169 41; (Gledening E D, Reed A E, Carpenter J A and Weinhold F NBO Version 3.1)

17. Frisch M J et al 2001 Gaussian 98, Revision A.11.2, Pittsburgh PA, Gaussian, Inc

18. (a) Foresman J B, Frisch A 1996 Exploring chemistry with electronic structure methods Pittsburgh, P A: Gaussian. Inc; (b) Jensen F 1998 Introduction to computational chemistry (New York: Wiley)

19. Koch U and Popelier P LA 1995 J. Phys. Chem. 99 9747

20. Popelier P L A and Bader R F W 1992 Chem. Phys. Lett. 189542

21. Perakyla M and Kollman P A 2000 J. Am. Chem. Soc. 1223436

22. Warshel A, Russell, 1986 J. Am. Chem. Soc. 108 6569

23. Daggett V, Schroder S, Kollman P A 1991 J. Am. Chem. Soc. 1138926

24. Staton R V, Perakyla M, Bakowies D, Kollman P A 1998 J. Am. Chem. Soc. 1203448 